TERRA. Revista de Desarrollo Local e-ISSN: 2386-9968

Número 9 (2021), 222-226

DOI 10.7203/terra.9.22100

IIDL - Instituto Interuniversitario de Desarrollo Local

\title{
Reseña. Emigrar después de la crisis. Crecimiento económico y nueva migración española
}

\author{
Susana Jiménez Guijarro \\ Graduada en Psicología y Máster en Migraciones Internacionales, Universidad de \\ Valencia (Valencia, España) \\ sujigui@alumni.uv.es
}

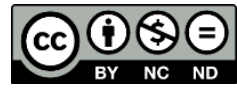

Esta obra se distribuye con la licencia Creative Commons

Reconocimiento-NoComercial-SinObraDerivada 4.0 Internacional 


\section{SECCIÓN RESEÑAS}

\section{Reseña. Emigrar después de la crisis. Crecimiento económico y nueva migración española}

Resumen: La obra aquí reseñada nos aproxima, de la mano de Ana Fernández Asperilla y Susana Alba, al que podría ser el primer trabajo centrado en el fenómeno migratorio sucedido tras la Gran Recesión (20082013) vivida en España. Además, las autoras tratan de romper con los mitos y clichés que alrededor de la temática migratoria se han forjado entorno a este periodo. Asimismo tratan de desmenuzar las condiciones sociolaborales y los rasgos de las políticas públicas con las que se encuentran los emigrados al exterior de España incluso en la actualidad, contexto temporal que podríamos caracterizar de relativa bonanza económica tras la crisis financiera iniciada en 2008.

Palabras clave: fenómeno migratorio, emigrar, Gran Recesión, alta cualificación, fuga de cerebros, retorno.

Recibido: 07 de diciembre de 2021

Devuelto para revisión: -

Aceptado: 09 de diciembre de 2021

Referencia / Citation:

Jiménez, S. (2021). Reseña. Emigrar después de la crisis. Crecimiento económico y nueva migración española. TERRA. Revista de Desarrollo Local, (9), 222-226. DOI 10.7203/terra.9.22100 


\title{
Ana Fernández Asperilla y Susana Alba
}

\section{EMIGRAR DESPUÉS DE LA CRISIS. CRECIMIENTO ECONÓMICO Y NUEVA MIGRACIÓN ESPAÑOLA}

\author{
Madrid (España). Fundación 1ero de Mayo, y Catarata, 2020. 208 pp.
}

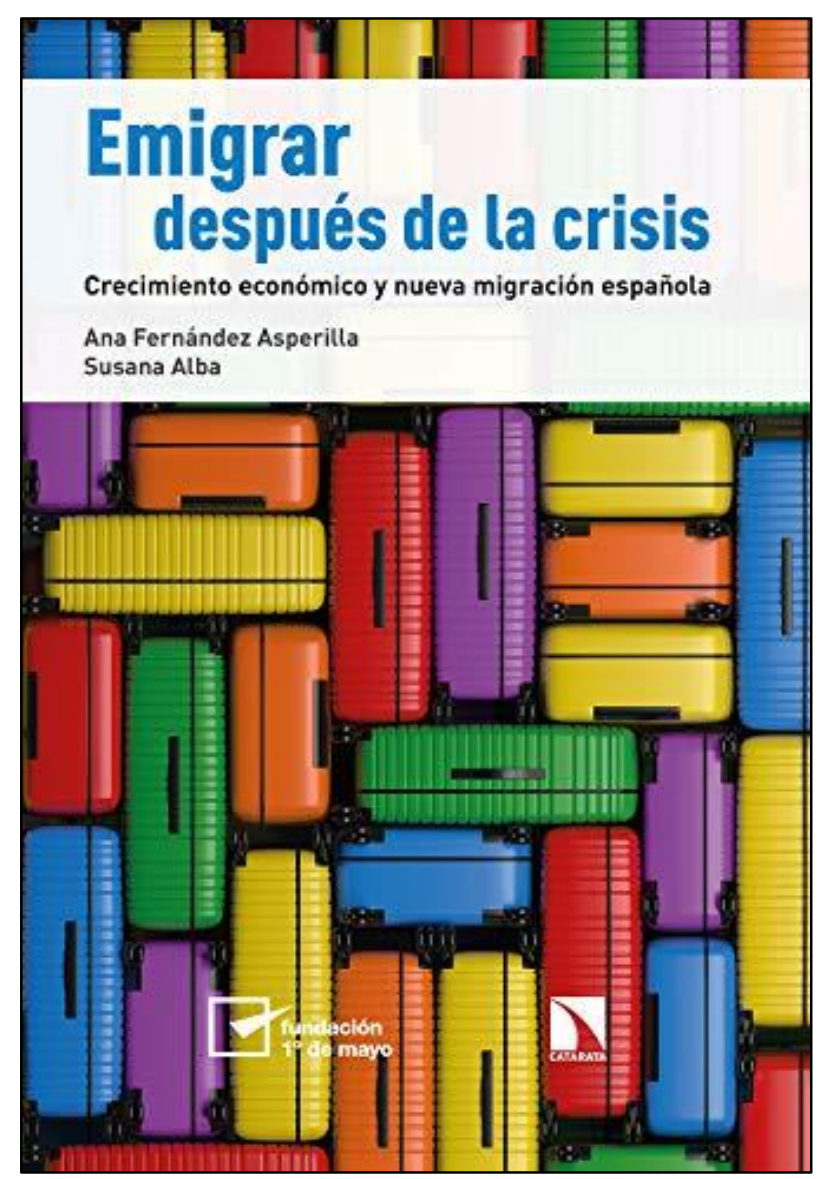

Las autoras de esta obra, Ana Fernández Asperilla, Doctora en Historia Contemporánea, Diplomada en Magisterio y Directora del Centro de Documentación de las Migraciones (CDM) de la Fundación $1^{\circ}$ de mayo, y Susana Alba, Licenciada en Geografía e Historia con Postgrado de Archivista y Documentación, y encargada del tratamiento técnico de los fondos documentales y colecciones del CDM, son dos profesionales de referencia en el ámbito de las migraciones que han sabido elaborar este estudio con su correcto análisis, interpretación y redacción de los datos obtenidos en diversas fuentes oficiales $y$ complementarias (como plataformas digitales de búsqueda de trabajo), respecto al fenómeno migratorio vivido durante la Gran Recesión es decir, a causa de la crisis del 2008.

$\mathrm{Si}$ existe un tema más controvertido debido a su relativo desconocimiento y a las innumerables malversaciones que se llevan a cabo en pos de la manipulación en favor de alguna que otra posición política, es sin duda el de las migraciones. Como este hecho es bien conocido entre aquellos familiarizados con el tema, nos encontramos con la necesidad de elaborar una obra rigurosa que resulte, a la vez, accesible tanto para los profesionales como para los lectores curiosos y, sobre todo, para que no quede impune ningún mito infundado o apoyado en datos poco fiables, pues si algo es de reconocer es que las migraciones constituyen una esfera de estudio compleja en lo que a recopilación de datos se refiere, ya que las fuentes que proporcionan dicha información no siempre resultan de fácil acceso y, del mismo modo, no siempre ofrecen una información veraz, pues en muchos casos resulta complejo aparecer en ellas si no se lleva a cabo el correcto procedimiento burocrático de registro y alta, una vez fuera del país de origen por más de 90 días consecutivos. A partir de aquí, las autoras se han centrado en realizar el análisis más minucioso posible para poder estudiar adecuadamente el fenómeno migratorio vivido en España tras la mayor crisis económica, hasta el momento, del siglo XXI. Para ello, el libro compuesto por un total de 208 páginas se divide en nueve capítulos que podríamos encuadrar en tres subapartados de la siguiente manera: la emigración desde España; los derechos y leyes para las personas emigradas y, el retorno a España. 
El visionado más general sobre la situación socioeconómica que había antes, durante y tras esta nueva tendencia migratoria en España, nos queda reflejada y explicada en el primer capítulo "Datos básicos de la emigración (2014-2018)", donde se aportan datos sobre la situación poblacional y migratoria dentro y fuera del país, así como unas primeras pinceladas sobre el retorno de los emigrados y con ellas las primeras evidencias de que no se trata de un fenómeno cíclico en el cuál todo lo que entra sale y viceversa.

El segundo capítulo, "La inserción laboral de los emigrados españoles en los países de acogida" resulta, a nuestro parecer, de los capítulos más ilustrativos en lo que a ruptura de mitos se refiere puesto que se adentra en el perfil tanto de la persona emigrada así como de los puestos ofrecidos en los países de acogida. Es este apartado el que nos permite rebatir ese discurso tan sonado sobre la "fuga de cerebros" o la "huida de talento" que podía estar experimentando España, pues las estadísticas nos indican ideas contrarias al respecto. De hecho, en este capítulo es en donde se trata de enfatizar que la característica más definitoria de las personas emigradas en aquel momento, era que perteneciesen a la población activa del país oscilando entre los 30 y los 39 años sobre todo, por lo que en el contexto sociocultural en que nos encontramos diríamos que se trata de una población joven en edad de trabajar y casi indistintamente del sexo que tuviesen (53\% hombres y $47 \%$ mujeres). Ya deja entrever, con los datos que se presentan a lo largo del capítulo, que el nivel de cualificación no es un rasgo definitorio del perfil del emigrado ni tampoco de los puestos de trabajo ofrecidos en los países destinos de los flujos, pues resulta escasa la proporción de personas con alta cualificación que obtenían en el exterior un puesto ajustado a ese mismo nivel de preparación.

Por el contrario, sí que es de mencionar que nada tiene que ver el perfil y las oportunidades laborales que se dieron durante la última gran oleada de emigración del siglo $\mathrm{XX}$ frente a los que se han visto en esta, pues en general todo está un estadio más "desarrollado" y, en general, las personas están más preparadas que entonces. No obstante, esto no quiere decir que no sigan demandándose perfiles de nula, baja o media cualificación para la diversidad de puestos que podemos encontrar ofrecidos en el exterior. Es decir, en comparación a la emigración vivida en el siglo pasado, esta vez el fenómeno estuvo representado por personas mucho más cualificadas en general (abarcando todos los niveles de formación) y relativamente de más edad, pero no en posición respectiva dentro de la sociedad (sigue estando representado por la población joven y activa laboralmente).

Por otro lado, ya en los capítulos tres, cuatro y cinco nos encontramos con una comparativa entre el antes y el después de lo que podríamos denominar "los derechos del emigrado español", en la que se apercibe la pérdida cada vez mayor de estos mismos derechos y facilidades de acceso a bienes, servicios y ayudas que, en algún momento estuvieron contempladas para esta población emigrada, y que con la llegada de la Gran Recesión se fueron retirando al principio paulatinamente, y más abruptamente después. Comenzaron por los derechos laborales, que parecían quedar alejados del país (de origen), y estos continuaron con los derechos políticos y sociales que, hoy por hoy, a pesar de la distinta situación, no se han vuelto a contemplar ni a incorporar en la legislación oportuna.

Todo esto, con el aumento incesante de la emigración española (también denominada "movilidad internacional", con ánimo de enmascarar tal fenómeno), que durante un momento inicial de la crisis fue hasta incentivada por el Estado español, pues de hecho, dado que interesaba alentar dichas salidas del país, este interés se acompañó de buenas condiciones y proyectos que resultaron muy necesarios y atractivos como en el caso de la exportación de la sanidad, la creación de las aulas ALCE, la entrada en vigor de la Ley de Memoria Histórica o la Ley Electoral (antes de ser reformada en 2011). Paralelamente 
a la crisis, toda esta dedicación de fondos públicos se tornaría insostenible, al igual que la realización del seguimiento de toda la población emigrada así como de su descendencia de la que el Estado español no obtenía (directamente) beneficio alguno. En consecuencia, las diversas administraciones del momento aprovecharon para reducir la inversión destinada a esta parte de la población, entre otros recortes dispuestos. Podría decirse, de hecho, que la población emigrada española se encontró con una situación casi de desamparo por parte de su propio país de origen, de modo que entre las ausencias de apoyo externo y las pocas garantías laborales que les ofrecían, cuando la situación mejoró en el país muchos ni siquiera contemplaron la opción de volver a España, lo que entonces se tornó en un problema que tratar de solventar. Toda esta información se trata más en profundidad en los capítulos seis y siete del libro y, conjuntamente a estos, encontramos las alternativas propuestas para impulsar el retorno de la población emigrada, tanto a nivel estatal como autonómico, en los dos últimos capítulos del libro (octavo y noveno, respectivamente).

La importancia de conocer las propuestas para incentivar el retorno la encontramos en el análisis del modo en qué se han ido sucediendo las fuerzas políticas en cada momento y, con estas, las estrategias que han tomado distinta "voz" y dirección en función de los intereses promulgados, así como las esferas que han quedado más descuidadas o empequeñecidas en cada momento. Esta es una cuestión, al igual que otra muchas, que se aprecia de forma elegante y transversal en toda la obra.

En síntesis, podemos decir que se trata de un trabajo muy completo y novedoso en lo que se refiere a temática específica y el periodo tratado. Además, evidencia un amplio y completo uso de fuentes acreditadas, así como la presencia de datos fiables que acompañan muy acertadamente cada una de las afirmaciones y estimaciones plasmadas. La redacción resulta impecable y no excesivamente técnica, de modo que puede resultar totalmente comprensible para una importante proporción de lectores sin necesidad de que se trate de profesionales en migraciones. Las autoras, como ya era de esperar dadas sus otras obras por separado, denotan un amplio conocimiento y experiencia sobre el tema de las migraciones y, sobre todo, un arduo recorrido persiguiendo la ruptura de estos estigmas y clichés que venimos mencionando en líneas anteriores, y que tan conscientemente desmenuzan a lo largo de toda la obra.

Susana Jiménez Guijarro

Graduada en Psicología y Máster Internacional en Migraciones (Universidad de Valencia, Valencia, España) 